\title{
Traceability in Medical Devices Design \& Manufacturing
}

\author{
Joseph Neelamkavil, Ajit Pardasani, and Michael Kernahan \\ Integrated Manufacturing Technologies Institute \\ National Research Council Canada \\ London, Ontario, Canada N6G 4X8 \\ \{Joseph.Neelamkavil, Ajit.Pardasani, Michael.Kernahan\}@nrc.ca
}

\begin{abstract}
This paper presents a methodology for establishing traceability between different types of information that evolve during medical devices development, and enable a complete information trail and knowledge management during the actual design and manufacturing process. The approach has been implemented in a Knowledge Management prototype that aims to help users capture, manage and reuse engineering knowledge during the various stages of product development. The methodology is illustrated by describing briefly with the system architecture and functionalities of the prototype system. The importance of traceability in helping designers to be more productive in reusing and adapting available design knowledge is also shown.
\end{abstract}

\section{Introduction}

To protect consumer's health and safety, regulations governing medical devices exist universally in all countries. All medical devices sold in a country must be in compliance with the local regulations. These regulations require that the medical device design and manufacturing processes be formally planned and documented, and that designs should be evaluated regularly throughout the development process. The rationale behind those regulations is that a good proportion of medical device recalls may be attributed to faulty product design. Though regulations greatly enhance the quality and reliability of the resultant medical devices, their execution adds overhead costs especially for new high-risk categories. To shorten the time from the ideation stage to the regulatory approval and market release, a computer-assisted product development tool that supports users to track related product development information would be extremely useful. Such a tool should provide the necessary support and guidance to manage the medical devices development process by offering the functionality to track the evolution of information generated during the medical devices development, manufacturing, testing and service, for example: a) The knowledge and rationale leading to design, manufacturing and testing decisions, b) Evaluation of the impact of design decisions against requirements and specifications, and c) Full information trail and knowledge management during the complete product life cycle, and d) Advice on the compliance to regulatory requirements and best practices.

Traceability refers to tracking the evolution of requirement of a product (e.g.: a medical device) from its origin, and following links of the various life-cycle entities created exclusively to satisfy the needs of the original requirement. A traceability system ensures hierarchical (parent-child) relationship that is typical in product assemblies, and this capability is essential in modeling designs at multiple level details. Using this feature, complex customer requirement can be broken down into sub requirements recursively. Traceability provides a mechanism to ensure that the original requirements are met, and gives rationale for the creation of downstream life cycle elements (e.g. specifications, design, test results, etc.). It can show how a change in the original requirement impacts on the downstream life cycle elements. Conversely, it can also show how a change in the physical system impacts the original requirement. A design entity is considered to be traceable if one can find why it exists, and it can be traced to other related design entities and information in the system. For example, a design concept is considered to be traceable if one can discover why this design concept was developed, what product function(s) are being met by it, and what detailed design and system components implement the conceptual design.

Systems that support the traceability help improve the product quality by enabling designers find out if all customer requirements are met. Traceability is essential in the design of safety critical systems such as medical devices to ensure that the government set standards is complied. A need for compliance to regulatory requirements and knowledge management demands that the design processes be formally planned, documented and traceable. Traceability also assists designers in giving them the opportunities to reuse the existing design knowledge by providing 
them with mechanisms to quickly trace the evolution of design solutions from the requirements.

\section{Product Traceability and Regulatory compliance}

Traceability requirements are woven into various Government regulations and international standards $[1,2]$. For example, an extract from ISO 13485:2003 states: "The organization shall establish documented procedures for traceability. Such procedures shall define the extent of product traceability and the records required... where traceability is a requirement, the organization shall control and record the unique identification of the product". ISO 14969: Medical devices Quality Management Systems - provides guidance on the application of ISO 13485: Medical devices quality management systems requirements for regulatory purposes, and 13488 Quality Systems Medical devices particular requirements for the application of ISO 9002. Section 4.4 of the ISO 14969 gives details on Design Control: "The extent of medical device evaluations, verifications, and validations should be commensurate with the nature of the risks and the benefits associated with the use of the medical device. Risk analysis using techniques such as fault tree analysis (FTA) and failure mode and effects analysis (FMEA) can be utilized at various stages of the design process..." Guidance is provided to many areas within the product development life cycle, including to those given in Table 1.

Health Canada has instituted regulations related to medical devices [3]. Canadian regulations are essentially based on four main aspects: Pre-market review of submitted data, Quality system (ISO 13485:2003), Post-market surveillance, and Risk based classification for the medical devices. Risk based classification involves 4 classes: Class I with no requirements at all, whereas Class IV devices need to comply with basically all requirements. The premarket review makes an evaluation on how complex the product actually is; it also requires elaborate documentation for risk class III and IV devices. It is to be noted that only product distribution and implantable devices traceability is covered by the regulations, whereas traceability associated with the manufacturing process is a direct requirement of ISO 13485.

\section{Table 1. Elements of 'Design Control'- source: ISO 14969}

\begin{tabular}{|c|c|}
\hline $\begin{array}{l}\text {-Input Requirements } \\
\text { The design inputs should be specified to the level } \\
\text { necessary to permit the design activity to be carried out } \\
\text { effectively and to provide a consistent basis for design } \\
\text { decisions, design verifications and design validation. } \\
\text { Examples of design inputs should be defined, } \\
\text { reviewed, approved by the supplier, include: }\end{array}$ & $\begin{array}{l}\text {-Design Review } \\
\text { Involving staff having no direct design responsibility } \\
\text { enables the reviewers to take into account all aspects of } \\
\text { the supplier's interest, e.g. manufacturing, marketing, } \\
\text { design, after-sales servicing and support, and likely the } \\
\text { medical effectiveness of the design. For medical devise, } \\
\text { design reviews should also consider the following: }\end{array}$ \\
\hline $\begin{array}{l}\text {-Intended clinical use } \\
\text {-Intended device performance and limits } \\
\text {-Performance during normal use, storage, handling, } \\
\text { maintenance } \\
\text {-Specifications for labeling; } \\
\text {-Instructions for servicing } \\
\text {-Environmental, safety and regulatory requirements } \\
\text {-Ergonomics and human factors } \\
\text {-Other standards } \\
\text {-Output spec. } \\
\text { Design outputs should include specifications for: } \\
\text {-Raw materials } \\
\text {-Component parts, sub-assemblies, finished devices } \\
\text {-Product and process software } \\
\text {-Quality assurance procedures \& acceptance criteria } \\
\text {-Manufacturing and inspection procedures } \\
\text {-Packaging and labeling } \\
\text {-Identification and traceability procedures } \\
\text {-Installation and servicing procedures }\end{array}$ & $\begin{array}{l}\text {-Has risk analysis been carried out to ensure safety } \\
\text { considerations? } \\
\text {-Is the labeling adequate? } \\
\text {-Will the design accomplish the medical use intended? } \\
\text {-Is the packaging adequate, particularly sterile device? } \\
\text {-Is the sterilization process adequate? } \\
\text {-Is the device compatible with the sterilization method? } \\
\text {-Design Changes } \\
\text { Whether or not the device is currently on the market, the } \\
\text { following considerations, among others, should be } \\
\text { addressed before permitting a change to an approved } \\
\text { design: } \\
\text {-Will the product still conform to agreed upon } \\
\text { requirements \& spec? } \\
\text {-Will the intended use be affected? } \\
\text {-Will different components of the product affected? } \\
\text {-Will there be a need for interface design (e.g.: physical } \\
\text { contact with other components)? } \\
\text {-Will the change create problems in manufacture, usage? } \\
\text {-Will the design be still verifiable? } \\
\text {-Will the change affect regulatory status of the product? }\end{array}$ \\
\hline
\end{tabular}


Table 2. Traceability - Record Keeping

\begin{tabular}{|l|l|}
\hline \multicolumn{2}{|c|}{ Traceability - Record Keeping } \\
\hline $\begin{array}{l}\text { Product Identification } \\
\text { (e.g.: serial number) }\end{array}$ & $\begin{array}{l}\text { Special inspection } \\
\text { requirements }\end{array}$ \\
\hline $\begin{array}{l}\text { Workstations involved } \\
\text { in production }\end{array}$ & $\begin{array}{l}\text { Acceptable quality level } \\
\text { information }\end{array}$ \\
\hline $\begin{array}{l}\text { Worker IDs and } \\
\text { Operations \# }\end{array}$ & Reject information \\
\hline $\begin{array}{l}\text { Date \& Time work } \\
\text { performed }\end{array}$ & $\begin{array}{l}\text { Repair/rework } \\
\text { information }\end{array}$ \\
\hline $\begin{array}{l}\text { Manufacturing batch \& } \\
\text { lot \# }\end{array}$ & $\begin{array}{l}\text { Environmental test } \\
\text { information }\end{array}$ \\
\hline $\begin{array}{l}\text { Product functional test } \\
\text { information }\end{array}$ & $\begin{array}{l}\text { Embedded software } \\
\text { information }\end{array}$ \\
\hline Quantity accepted & $\begin{array}{l}\text { Packaging/Shipping } \\
\text { information }\end{array}$ \\
\hline Quantity rejected & $\begin{array}{l}\text { Returned item } \\
\text { information. }\end{array}$ \\
\hline
\end{tabular}

US Food and Drug Administration (FDA) have imposed elaborate requirements - Quality system regulations - pertaining to medical devices. FDA requires manufacturers to identify parts and assemblies during each stage: receipt, manufacturing and distribution. The rationale is that it can minimize the impact of product recall, which may become necessary if a defect in any part or assembly is discovered. There also exist regulations pertaining to current Good Manufacturing Practices (cGMP). Some aspects of these regulations are: They are more stringent for higher risk devices (example: implants). The design/development processes must include "objective evidence" that the design output meets the design input requirements; production and process controls demonstrate that the device conforms to specifications; if the manufacturing process could cause deviation from specifications then process controls must be established, coordinated with acceptance activities; prototype production units and variations of existing designs need to be tested under actual or simulated use conditions (bench testing and cellular/animal studies). Though slow, tedious and expensive processes (implants, surgical, interventional instruments, imaging technologies), they are required, and records need to be available/submitted for inspection. Similarly, to maintain that products have met all design requirements, device history records and device manufacturing records must be kept [4]. Forms such as the one shown in table 2 can be designed and used to capture details of the many tasks associated with various stages of the product life cycle, which can be stored in a database. This can accelerate the certification process, while at the same time the design and implementation of the traceability system become a lot easier.

\section{Traceability during Medical Devices Development}

Traceability in medical device development refers to information modeling of various information entities applicable to the device life cycle e.g. requirements, design, manufacturing, testing, implant, and monitoring. Since design activities are driven by requirements, these provide reference criteria against which the performance of the design solutions is evaluated, verified and validated. Device functions are derived from the customer requirements and specifications by analyzing "what a device must do" in a solution-independent way. The functions are then realized by implementation of design concepts (working principles) into a physical system structure. All the information generated through the product development process is interlinked so one is able to trace the evolution of requirements to the device components and all the intermediate stages as shown in Figure . Documenting the design evolution of a device ensures forward traceability to verify requirement compliance, and backward traceability to analyze effects on requirements if a system solution is changed.

Traceability systems play an important role for products that have to meet the government set standards for safety. Especially, medical devices have to satisfy regulatory requirements to demonstrate the safety of the product. A significant part of a regulatory submission requires sufficient documentation to confirm that the manufacturer has conscientiously implemented an appropriate process for the design, testing, production, and quality assurance. Traceability systems enable capturing the documentation trail for regulatory submission.

Though requirements can be handled with text documents this method has limitations to being useful for simple product structures and smaller product development teams. In text documents, relationships between different information entities can be captured through cross-referencing and hyper linking. This method may prove to be adequate for simple products but does not provide an effective solution for browsing and searching traceability information for complex products like medical devices. Hence, a well-designed data schema, system architecture, and user interface are central to the realization of benefits from traceability systems. 


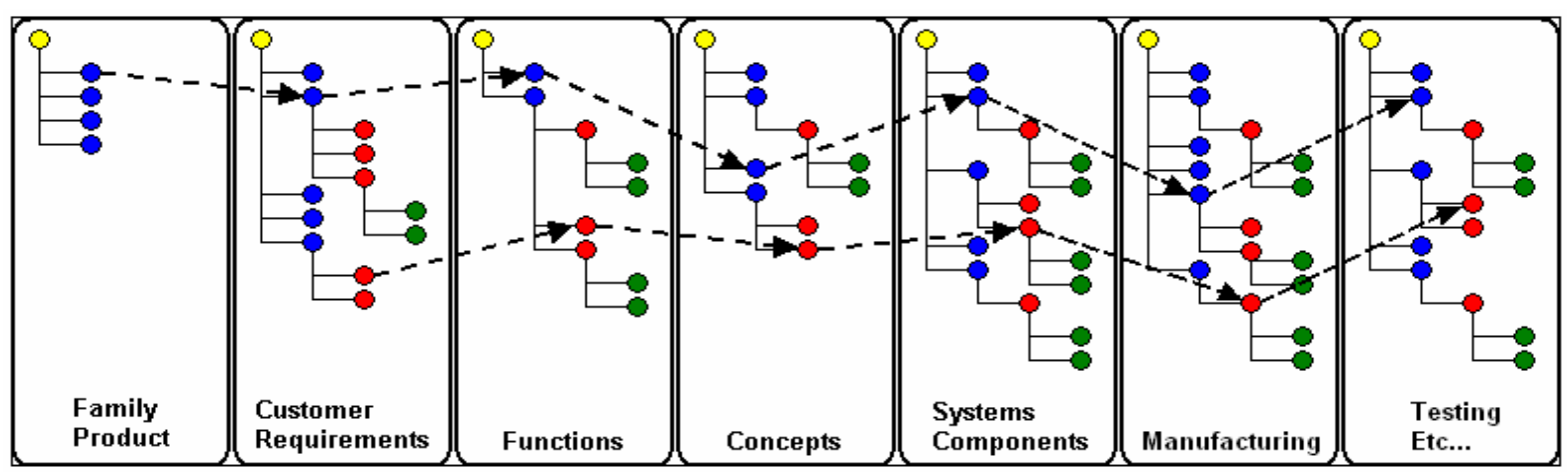

Figure 1. Traceability of information from one lifecycle domain to

\section{Systems Characteristics for medical device life cycle data traceability}

Let us now look into how the regulatory compliance can be made easier with the aid of a software system. It is desirable that the system provides support for the dynamic traceability, which is the ability of the system to update and maintain traceability information at the most current level. Systems with static traceability do not update with the evolution of design hence lose their usefulness when the design undergoes changes. Though the systems that have the dynamic traceability require constant effort to maintain, they remain relevant over the time.

Another desirable characteristic of traceability system is to provide bi-directional trace that is different from the child-parent traceability. Whereas the bidirectional traces allow for links between the entities of different domains, the parent child relations is desirable for capturing nesting/containment relationships and provides the capability to model a design entity at multiple hierarchical levels. This capability is useful to model complex design entities at a multiple level of details. Using this capability, complex customer requirement can be broken down into sub requirements recursively. Parent child traceability also lets modeling relationships of parts/components to an assembly.

User Interface plays a crucial role in determining the overall effectiveness of traceability systems. If the user interface is easy to navigate, people will probably use the system [5]. An easy to use browsing mechanism will let the user view, trace and search requirements and related life cycle information. The user interface should also allow for easy entry, and structuring of information and at the same time provides mechanisms so that user is able easily establish traces between various elements.

Traceability systems must be able to let users find how device requirements are implemented; analyze the impact of changing a requirement; find what design decisions affect the implementation of a requirement; analyze the impact of changing a design solution on the requirement; find what systems and components are instrumental in realization of requirements; find test documents to be used to verify a requirement.

\section{Traceability Knowledge Management System Functionality}

In this section we describe the traceability features of the Knowledge Management Environment that has been developed at the Integrated Manufacturing Technologies Institute [6, 7]. The system enables designers to capture the knowledge that arises during the design process, to structure such knowledge, and find the desired design knowledge easily. It allows users to link interrelated pieces of product information such as products to customer requirements, customer requirements to related product functions, functions to design concepts, concepts to assembly systems/components, etc. The relationships between various design entities are established by following organization's practices for documenting traceability information. Through the analysis of available documents, one can trace what customer requirements contributed to which functional specifications and eventually to what physical systems/components in both forward and backward directions. Once the system has been populated with traceability information, it can reveal the impact of requirement changes that result in design modifications and vice versa. The system also supports hierarchical or parent-child relationships among requirements, functions, and so on. In general, the prototype system provides the capability to model the information that is generated during the various design stages; it provides functionality to store, search, retrieve and browse through the vast amount of information pertaining to design.

The system presents a knowledge base as a series of columns, as shown in Figure 2. It provides easy 


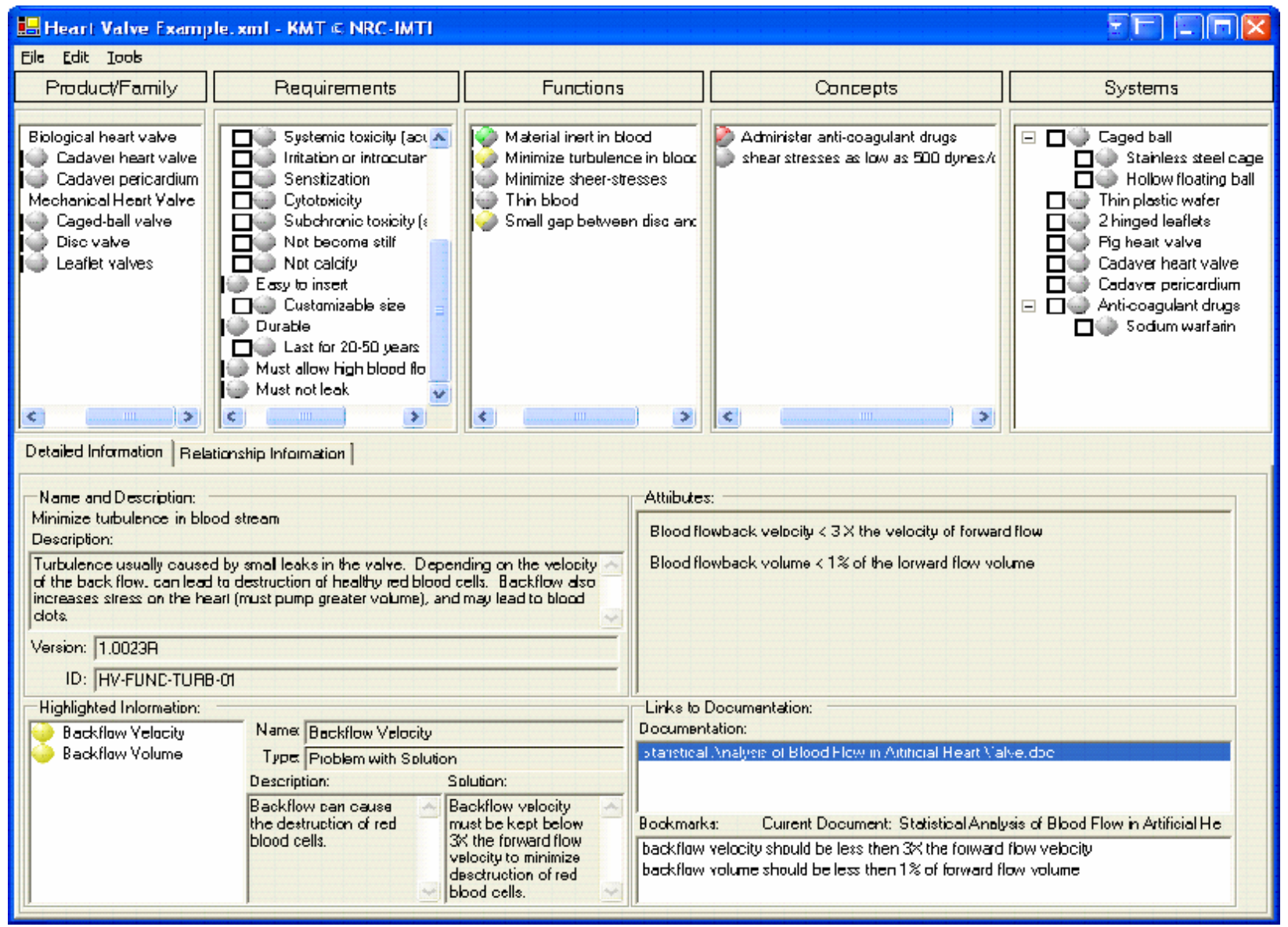

Figure 2. Design Browser showing structured design information of several heart valves. The browser shows high-level design information at a glance. Its columns correspond to various design phases. The text view in the lower part of the window shows design attributes of design entity that has been selected in the column above.

navigation capability through the existing knowledge base to locate desired design knowledge. The design browser shows the evolution of design information from customer requirements to the complete product structure at a glance. In our example, the associated information is organized under five columns: Product Type, Customer Requirements, Functions, Concepts and System Structure. The Product Type column is titled Product/Family and contains the names of products and associated product families. The Customer Requirements column consists of one-line descriptions of the customer needs. The Function column contains brief descriptions of what the product does. The Concept column has information on how the product does its functions, and finally the System column depicts the product structure through hierarchical relationships among assemblies and associated components. The browser helps designers in finding the inter-related design information through a traceability feature. This enables users to map and link an entry in a column to multiple entries in any of the columns. A user can link a customer requirement to one or several functions that satisfy that requirement. Several requirements may be met by a single function.

The interface we have created allows the designer(s) to be able to quickly identify all of the affected entities. Checking off a single entity will cause the other node lists (in one or both directions) to show only those entities that are directly or indirectly related to that entity. Taking the requirement example from above, checking off an individual entity in the requirements column will leave only the functions that are directly related to the entities or its parents or children. The concepts column is updated to display only concepts that are related to the displayed functions (therefore indirectly related to the requirement). This is continued down to the systems and components level. The interface also allows reverse traceability, so a system can be checked in order to evaluate what concepts it is related to, all the way up to the requirements (and product) that it is indirectly related to. 


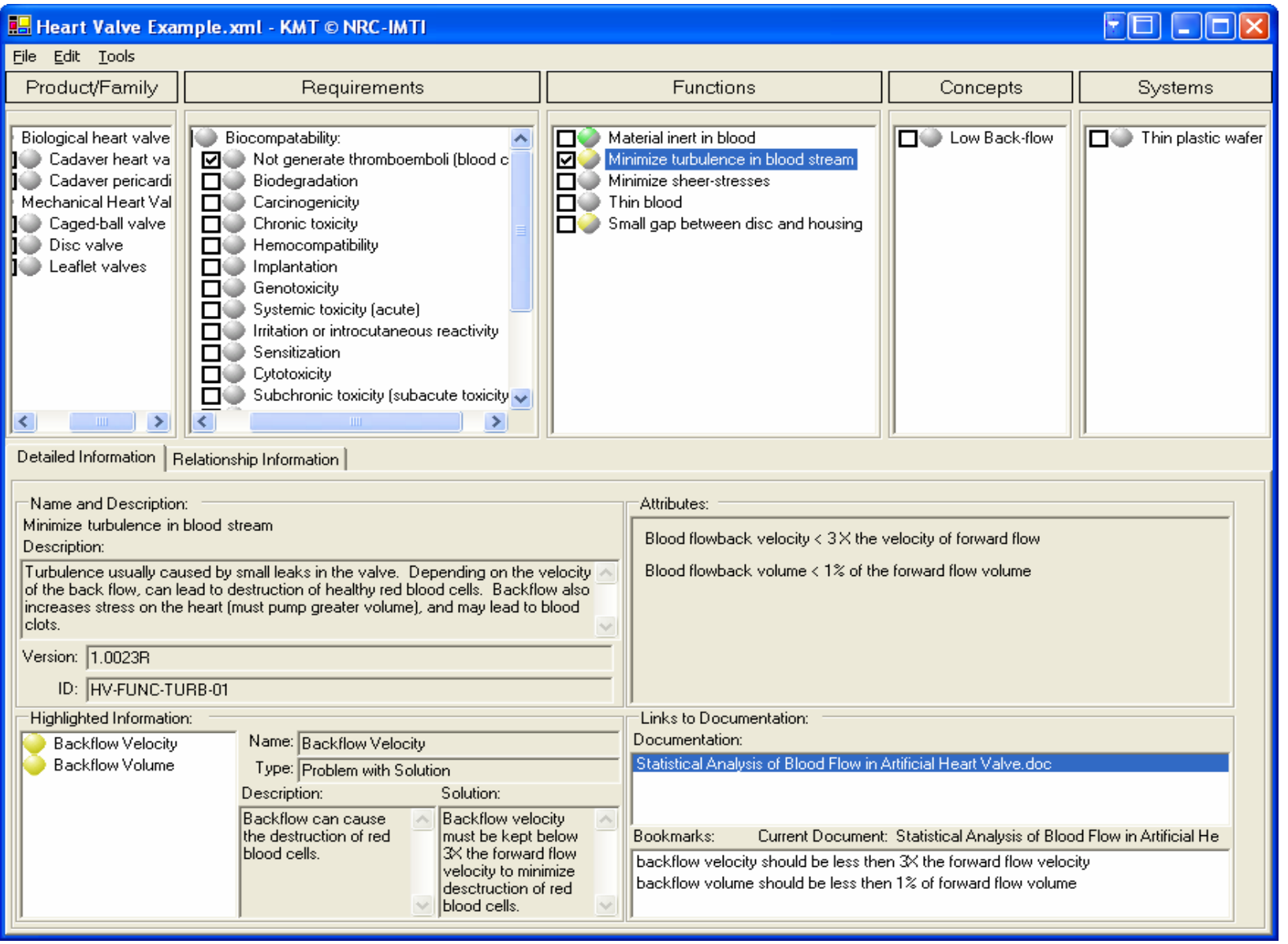

Figure 3. Design Browser showing the traceability relationships between the selected Requirement and the associated design entities in the Function, Concept and System columns.

Selecting an entity from any of the columns will display detailed information about the entity in the bottom portion of the interface. Foremost is a detailed description of the entity, where the designer can explain the entity in detail, as well as any version information and an ID for the entity. Note that, when medical devices are manufactured, its design version, product serial ID, location of usage (patient ID if it is an implant), etc. need to be tracked. The attributes section can be used to list out important measures and values that relate to the entity. The highlighted information section allows the designer to tag entities with flags of various degrees, from important information (green), to a problem with a solution (yellow), to a problem without a solution (red). These flags are communicated to the user visually by the multi-colored balls in the browser interface. Documents can also be linked to individual nodes, as well as bookmarks within the documents. This is useful for providing links to documents that influenced and document the decisions made by the designer.

The functional capability of the knowledge management system is illustrated for the design of an Artificial Heart Valve. Figure 3 shows all functions, concepts and systems that are related to the "Not generate thromboemboli (blood clots)" requirement which is met by several functions including "Minimize turbulence in blood stream." This function is also checked, so that only those Concepts and Systems related to it are displayed, pruning the trees down to "Low Back-flow" related to the "Thin plastic wafer" System.

When creating a new design from scratch, the customer requirements for the new design are structured in hierarchical format within the requirements category of the system. The requirements can be nested as well as linked to the product that is currently being designed. Once customer requirements have been captured, associated functions that fulfill the requirements are added to the system. These functions can also be hierarchical. As more and more requirements and functions are added to the product model, the richness of the traceability within the product grows deeper and deeper. Similarly, concept and system component information 
is added to the design model and linked appropriately within the traceability model. Components are added as parts of systems as opposed to having their own dedicated column in the Design Browser. This approach was taken, as the traceability information for any individual component will be back to the system that it is a part of.

\section{System Architecture}

This system was created in the Windows environment using the C\# programming language in Visual Studio 2003. Figure 4 describes the architecture of the system. The data model stores the design information and allows the browser and editor access to the information. XML is used as a format to save the traceability information as it can be parsed easily and integrated with external systems. The Browser and Editor are both event-based systems, meaning that changes in the data model are instantly reflected in them. The XML enables the separation of content from style thus facilitating multiple views of same data.

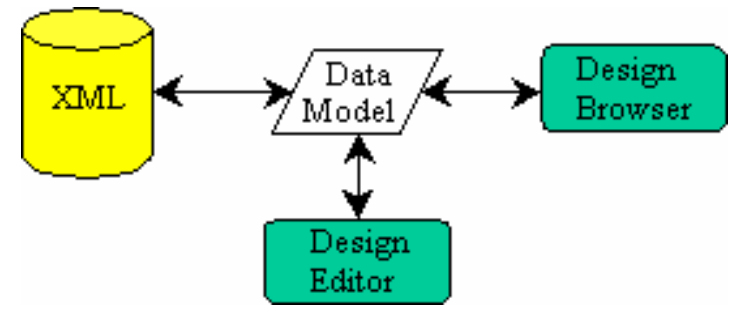

Figure 4. System Architecture

\subsection{XML Data Model}

Figure 5 shows the major aspects of the XML schema that supports the traceability features by enabling referencing from one design entity to another. The root of the XML tree is an element "KnowledgeBase." The "KnowledgeBase" contains three sub-elements, which encapsulate all of the design knowledge.

The first sub-element is the "KnowledgeTypes" element. This element encapsulates all of the information about the different types of knowledge, such as "Requirement" or "Function," as well as links to their top level Knowledge Objects.

The second sub-element is the "KnowledgeObjects" element. This element encapsulates all of the different knowledge objects that are displayed in the columns in the traceability interface. Each knowledge object also contains elements related to any important information, any documentation, and any attributes that have been specified.

The third sub-element is the "LinkTypes" element. This element contains all of the different types of links that exist in the knowledge base. Each relationship between two elements in the knowledge base has a link type, and an instance of this relationship is called a "LinkObject." A link object knows what type it is, as well as pointing to a parent and a child knowledge object.

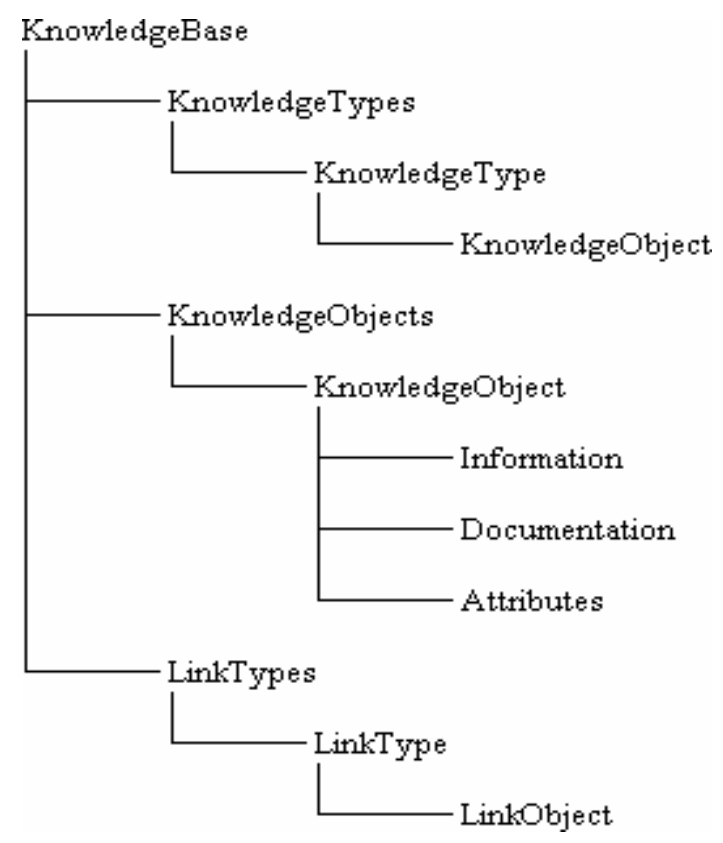

Figure 5. XML Data Model

\section{Summary}

The paper has described traceability features of the Design Knowledge Management System during medical devices development. The traceability features of the system allow users to study the impact on changing requirements on upstream/downstream design entities thus enabling a full information trail and knowledge management during various phases of the design. The system accelerates the product development process by helping reuse of prior design knowledge in new design situations. A system that establishes the traces between requirements, and all information generated throughout the product development cycle will help medical device manufacturers to plan, manage and reuse design knowledge in a very efficient manner. In a nutshell, it can

- Caution on the impact of design decisions (engineering changes, material changes, product certification) on customer requirements, which 
will contribute to design efficiency and faster decisions.

- Ensure forward traceability to verify requirement compliance, and backward traceability to assess the impacts on requirements when a design solution needs revisions.

- Provide full information trail and knowledge management during product development and advice on the compliance of regulatory requirements and company best practices.

\section{References}

[1] ISO 13485, 13488, 14969 - Quality Systems Medical Devices

[2] K. Stinshoff, "Expectations for compliance with the traceability requirements of the EU IVD Directive and associated ISO Standards", AACC 2003 Annual
Meeting, Philadelphia, USA 2003.

[3] http://www.hcsc.gc.ca/english/about/acts_regulations.html

[4] J. Schultz, "Component and Assembly Traceability in Medical Electronics Manufacturing", Medical Electronics Symposium, 2004.

[5] D. Svensson, J. Malmqvist, "Integration of Requirement Management and Product Data Management Systems", Proceedings of DETC'01, ASME 2001 Design Engineering Technical Conference, Pittsburgh, PA, September 9-12, 2001.

[6] J. Neelamkavil, A. Pardasani and M. Kernahan, "Knowledge Management System for Early Design", International Conference on Information and Knowledge Engineering, IKE'02, Las Vegas, USA. , June 2002, pp: 184-190.

[7] J. Neelamkavil, M. Kernahan, "A Framework for Design Knowledge Reuse", ASME International DETC/CIE conference, Chicago, September 2003. 